\title{
Justification of parameters of wheelset axle fatigue strength test-bench for railway rolling stock
}

\author{
Oleksii Kuropiatnyk ${ }^{1, *}$, Serhii Raksha ${ }^{1}$, and Pavlo Anofriev ${ }^{1}$ \\ ${ }^{1}$ Dnipro National University of Railway Transport named after Academician V. Lazaryan, Department of Applied Mechanic and \\ Material Sciences, 2 Lazaryana St., 49010 Dnipro, Ukraine
}

\begin{abstract}
The operation safety of the railway rolling stock depends directly on the strength of the base part of the wheelset - its axle. Therefore, in recent years numerous studies of theoretical and experimental nature, both for means of rail transport, and for machines containing rail track equipment are carried out. This paper substantiates the main parameters of the fatigue test bench for axles for wheelsets of railway rolling stock. We performed an analysis of the load schema for bench tests, determined the dependence of the bench work force on the parameters of the wheelset axle. In addition, we substantiated the design and parameters of vibrator. For the research, we adopted the following criteria of rationality: work force, energy consumption and longitudinal dimension of the test bench. The design is considered the best, if these criteria acquire the smallest values. The results of the research indicate that the load scheme "cantilever beam" is rational for the fatigue bench tests of the axles for wheelsets of railway rolling stock. The best design of the vibrator have to provide the test bench work force by rotating the unbalanced mass around the stationary wheelset axle. The rational vibrator contains a sectoral eccentric mass rotating at an angular velocity of about $150 \mathrm{~s}^{-1}$. In this case, the eccentric mass value is about $80 \mathrm{~kg}$, and its eccentricity is $135 \mathrm{~mm}$.
\end{abstract}

\section{Introduction}

In many industries, the widespread used was gained by railway transportation vehicles. They are used for transportation loads and people within the precincts of an enterprise, open-pit mine or in mines (intra-company industrial conveyance facilities) or ensure traffic between certain enterprises (external industrial conveyance facilities, including the railway transport).

Each of railway transportation vehicles includes a bogie that contains, in particular, a wheelset (WS). This component part containing an axle as a basic part, running wheels (or a single wheel for semi-axles) and a gear wheel (for driving axles).

The operational safety of a railway transportation vehicle is directly related to the strength of wheelset basic part, i.e. its axle. Therefore, latterly numerical studies of theoretic and experimental nature are conducted, both for railway transport vehicles [1, 2, 3], and for motor vehicles that contain railway running equipment $[4,5,6]$.

Before assembly of a wheelset certain number of axles from a batch is subjected to testing. For this purpose, special equipment is used, in particular, testbenches that imitate application of forces equivalent to operational loads.

In accordance with interstate standard [7] the following types of bench tests were established for wheelset axles:
- Testing when loaded with static vertical and lateral horizontal forces;

- Testing when loaded with tangential forces of threshold adhesion values with consideration of wheelslip;

- Fatigue strength testing when loaded with cyclic rotational bending.

The latter of the types of testing is the most complex because it renders operation of the bench under conditions of significant dynamic loading. Quite a number of studies were conducted on the issues of establishment of a rational pattern or loading, determination of design and performance. Results of such studies got their reflection in a number of safety documents $[8,9,10]$, and in the standards database $[11$, 12].

The analysis of information sources showed that the problem of a rational design development and justification of wheelset axles fatigue strength test-bench parameters for railway transport is a pressing one, but still remains unsolved.

The purpose of this work is to justify basic parameters of wheelset axles fatigue strength test-bench for railway transport.

In order to accomplish a determined objective the following problems require solving:

1) Justification of a rational pattern of loading under bench testing;

2) Identify dependence of test-bench operational loading from parameters of wheelset axle being tested;

Corresponding author: kuropyatnick@gmail.com 
3) Justify the design for vibrator, i.e. sources of testbench operating force;

4) Determine parameters of the vibrator.

For the purpose of obtaining and analyzing the results, the methods of mathematical modelling were used involving special-purpose software complexes.

\section{Justification of a rational pattern of loading}

General requirements to conducting wheelset axle fatigue strength bench testing are set out in the interstate standard [11]. According to this standard the wheelset axle is stipulated to be set on two hinged supports, one of which is to be set as close as possible to the box journal face, and the other one - at the section between surfaces of running wheels installation (Fig. 1, a). Therewith, test-bench operating force $\left(F_{1}\right)$ acts in radial direction on the surface of the running wheel installation located between supports. In such a case the pattern of loading can be presented as a beam on two hinged supports with a transverse concentrated force between them (Fig. 1, b). The standard does not regulate the magnitude of this force, and beam sizes are indicated in relation to design and type of the axle.

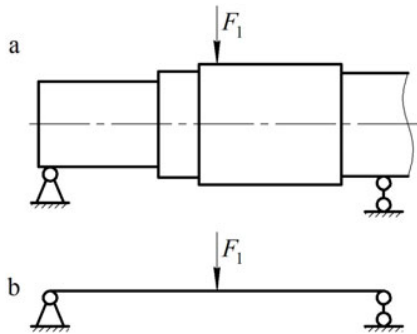

Fig. 1. Installation layout (a) and loading pattern (b) of wheelset axle as per standard [7]

Another interstate standard [12] specifies that cyclic rotational bending of wheelset axle can be implemented through rotation of the imbalance weight installed on the box journal (location of hinged stationary support in Fig. 1, a). Moreover, the axle can be press-fit into the running wheel (or into the temporary process hub installed instead of the wheel), fixed against shifting (Fig. 2, a). Under such conditions the pattern of loading represents a beam constrained from one end with its console under applied concentrated force $F_{2}$ (Fig. 2, b). The magnitude of this force, as in the previous case, is not regulated, and the dimensions of the pattern depend on wheelset axle sizes.

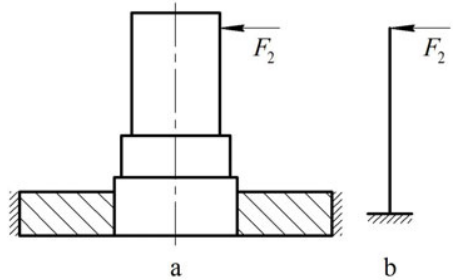

Fig. 2. Installation layout (a) and loading pattern (b) of wheelset axle as per standard [8]
It should be noted that in case of testing as per the pattern in Fig. 1 or Fig. 2 only a part of a wheelset axle is installed on the bench.

Another two patterns of axle loading should be noted that to a greater extent correspond to actual conditions of wheelset usage. Each of these is based on presenting the wheelset axle as a beam on two hinged supports. In one case the support points are located on surfaces of running wheels installation, and the test-bench operational force $\left(F_{3}\right)$ applied to box journals imitates the loading from the bogie (Fig. 3, a). The other pattern provides a location of support points on box journals with application of testbench operational force $\left(F_{4}\right)$ to running wheels installation surfaces thus representing an imitation of rail track force response impact to bogie action (Fig. 3, b).
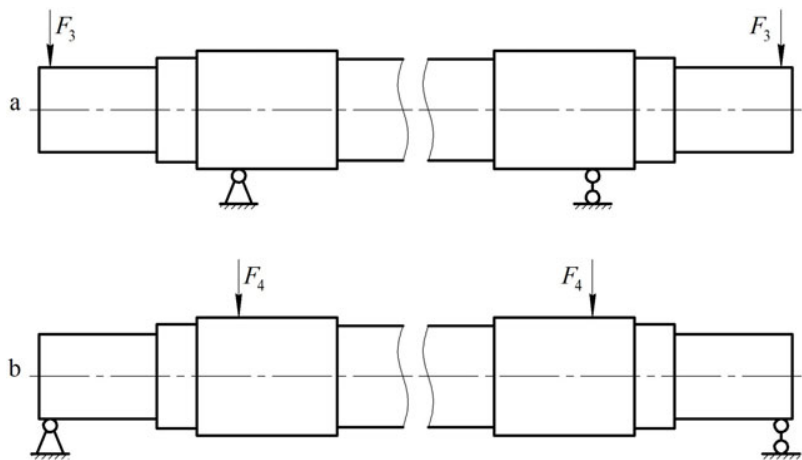

Fig. 3. Wheelset axle installation layouts approximated to service conditions

The common lack of both layouts as per Fig. 3 the requirement of testing axles as a whole that increases testbench overall dimensions as compared with layouts as per Fig. 1, 2. Therefore, let's hereinafter consider the first two layouts only:

- "Free-ended beam" (Fig. 1, b) - installation of wheelset axle on two hinged supports with application of the operational force radially to running wheels installation surface located between supports;

- "Cantilever beam" (Fig. 2, b) - fixation of wheelset axle using an intermediate piece (running wheel or temporary process hub) with application of test-bench cantilever operational force to the box journal

In order to justify a rational pattern of loading let's adopt the following criteria:

- Test-bench operational force (and, as an effect, power consumption);

- Overall dimension lengthwise.

The best one is the pattern, wherein both criteria gain lowest values.

\subsection{Comparison by operational force magnitude}

When conducting the fatigue strength tests the wheelset axle cyclic bending is implemented. Disregarding the loading pattern the bending stress in any cross-section is determined by the formula

$$
\sigma=M / W,
$$


where $M$ - bending moment in the design cross-section; $W$ - section modulus of the design cross-section.

Considering that the section modulus $W$ depends only on wheelset axle dimensions, the selection of a rational pattern of loading is made by the magnitude of the bending moment.

The bending moment is determined as follows:

1) For the "free-ended beam" pattern

$$
M=0.5 F_{1} x
$$

2) For the "cantilever beam" pattern

$$
M=F_{2} x .
$$

In these formulas $F_{1}$ and $F_{2}$ are the test-bench operational forces for the first and the second patterns of loading accordingly; $x$ is the coordinate of the design cross-section (the same for both patterns).

The formulas presented show that in case of gaining the same bending stress values the operational force as per the loading pattern 1 has to be twice as great as compared to the operational force of test-bench with the loading pattern 2. Therefore, by the criterion of the test-bench operational force the rational one is the "cantilever beam" loading pattern.

\subsection{Comparison by the test-bench overall dimension lengthwise}

The test-bench overall dimension lengthwise directly depends on geometrics of the loading pattern (beam). The same portion of wheelset axle is being tested. One end of the beam is the box journal face; it has one of hinged supports located thereon (for the "free-ended beam" pattern) or the cantilever loading is applied thereon (for the "cantilever beam" pattern). However, the distance from this beam end to the opposite one for different loading patterns is not the same. The other end of the cantilever beam (as a point) corresponds to one of crosssections of the running wheel installation surface on the wheelset axle, whereas for the free-ended beam this point is located further, between two running wheels installation surfaces. Thus, the length of such beam is greater, therefore by criterion of the test-bench overall dimension lengthwise the rational loading pattern is the "cantilever beam".

It follows from the above that by operational force criteria (and therefore, power consumption as well) and by test-bench overall dimension lengthwise the rational loading pattern is the "cantilever beam", for which the specified values will gain the lowest magnitude.

\section{Determination of a dependence of the test-bench operational force from wheelset axle parameters}

According to the formula (1) the bending stresses in the axle cross-section, described by the coordinate $x$, depend on two factors: bending moment that is determined by the formula (3) for the selected loading pattern ("cantilever beam"), and the section modulus of the design cross-section. Such a cross-section is the fillet from the box journal to the step between the journal and the running wheel installation surface (Fig. 4).

The section modulus of the design cross-section with the diameter of $d_{x}$ is determined by the formula

$$
W=\frac{\pi d_{x}^{3}}{32}
$$

Considering the nature of testing (fatigue strength testing) and particulars of cyclic loading implementation (by the symmetric cycle), let's adopt $\sigma=\sigma_{-1}$, where $\sigma_{-1}-$ axle fatigue limit under symmetric loading.

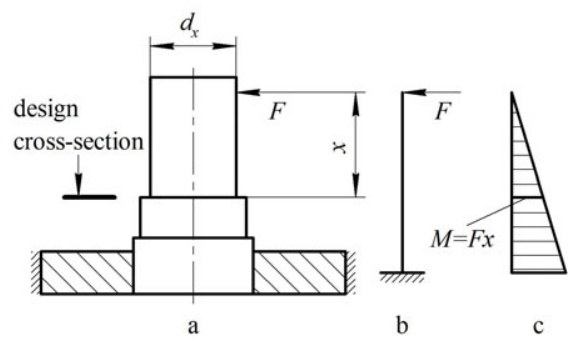

Fig. 4. The pattern for determination of the bending moment in the design cross-section: $\mathrm{a}$ - wheelset axle installation layout; $\mathrm{b}$ - loading pattern; $\mathrm{c}-$ distribution of bending moments

Through combination of the formulas (1), (3) and (4), we obtain:

$$
\sigma=\frac{M}{W}=\frac{32 F x}{\pi d_{x}^{3}}=\sigma_{-1}
$$

Therefore, the test-bench operational force is

$$
F=\frac{\pi d_{x}^{3}}{32 x} \sigma_{-1}
$$

It should be noted that the design cross-section diameter $d_{x}$ and the coordinate $x$ are wheelset axle characteristics that are regulated by the relevant standard [11]. The fatigue strength $\sigma_{-1}$ is determined as per the standard through testing [12], and therefore for each nominal size of wheelset axle the test-bench force $F$ can be determined in advance. The expression (6) also shows that when designing the test-bench intended for testing axles of several nominal sizes it is required to determine not only the geometry variability of specimens under test, but various magnitudes of the operational force as well.

\section{Justification of vibrator design}

Within this work let's consider as vibrator a test-bench element that creates an operational force and ensures its application to wheelset axle.

The test-bench cyclic operational force can be applied to wheelset axle in several ways:

1) Using a pulsator (variability of the force as per the harmonic law);

2) By means of rotating the imbalance weight (debalance) around the rigid wheelset axle; 
3) By means of rotating the wheelset axle with a debalance installed thereon;

4) By means of pressing with a test-bench working tool on the rotating wheelset axle while maintaining the constant magnitude of pressure corresponding to the amplitude of cyclic operational force.

Considering that the test-bench operational force has to ensure cyclic rotational bending of the wheelset axle as per symmetric cycle [12] we come to conclusion that the first way is the most complex one in the context of vibrator structural design.

Since the wheelset is much heavier than the debalance, the vibrator will consume more power in case of the test-bench operational force application in the third or the fourth way.

Hence, by the criteria of design complexity and power consumption the best one is the vibrator that ensures the test-bench operational force application by rotating the imbalance weight (debalance) around the rigid wheelset axle. In case of using it the criteria used gain the smallest magnitude. Let's assume it as a basis to carry out further studies.

The operating principle of the vibrator of chosen design is based on the fact that in the course of debalance rotation a force emerges being proportional to the weight $(m)$, eccentricity $(e)$ and angular speed $(\omega)$ of debalance rotation:

$$
F=m e \omega^{2} .
$$

The vector of this force is constant by magnitude, and the variability of its direction ensures the wheelset axle rotational bending.

The vibrator has the following components (Fig. 5): 1) motor; 2) elastic coupling; 3) multiplying gear; 4) gear-type coupling; 5) debalance.

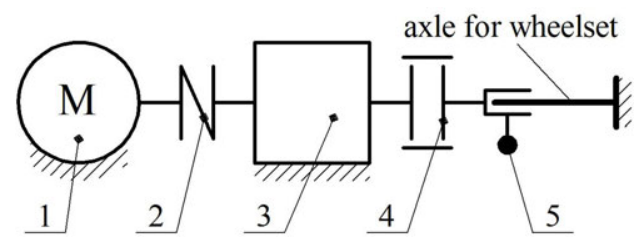

Fig. 5. Kinematic layout of the vibrator

Therewith, the multiplying gear may be optional; in such a case the duration of testing corresponding to the basis of testing [12] is significantly increased.

\section{Determination of vibrator parameters}

The basic element of the vibrator creating the test-bench operational force and determining its magnitude is debalance having the following parameters:

- Shape of debalance;

- Eccentricity of debalance (e);

- Weight of debalance $(m)$;

- Angular speed of debalance rotation $(\omega)$.

The first and the last characteristics relate to the number of debalance parameters rather conditionally: the debalance shape is impossible to be measured (however, it can be assigned a sequence number), and the angular speed $\omega$ depends on the motor shaft rotational frequency and the multiplier gear ratio (considering the independence nature of these values their direct influence on the value $\omega$ is numerically ambiguous).

Let's determine rational debalance parameters subject to adherence to a certain test-bench operational force $(F)$. The criterion of rationality is vibrator power consumption.

Weight $(m)$ and eccentricity $(e)$ of the debalance are linked by several dependencies:

1) Test-bench operational force; according to the formula (7)

$$
F=m e \omega^{2} ;
$$

2) Weight of debalance

$$
m=\gamma V(e),
$$

where $\gamma$ - density of the material, whereof the debalance is made; $V(e)$ - debalance volume against its eccentricity.

Upon the condition of $\left(7^{*}\right)$ the nature of debalance weight dependence from its eccentricity is hyperbolic. The debalance volume (and hence, the weight) are proportional to its eccentricity. Therefore, identification of dependencies $m(e)$ from expressions $\left(7^{*}\right)$ and (8) allows laying down parameters $m$ and $e$ for a debalance of any shape at a certain rotational frequency $\omega$.

For clear presentation of the procedure of determination of debalance rational parameters let's consider the test example. Test item - axle for freight wagons. Axle dimensions required for calculation [11] (Fig. 6):

- Box journal diameter $d_{1}=150 \mathrm{~mm}$;

- Box journal length $l_{1}=210 \mathrm{~mm}$.

Thickness of debalance will be taken equal to thickness of the bearing, whereon it will be installed. Due to presence of wheelset axle scenes during the test we will use a double-row barrel-type radial roller bearing with the following parameters:

- Internal diameter $d=d_{1}=150 \mathrm{~mm}$;

- External diameter $D=270 \mathrm{~mm}$;

- Width $B=73 \mathrm{~mm}$.

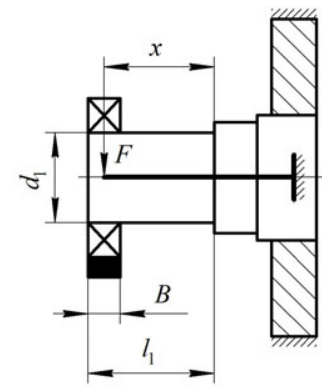

Fig. 6. Fragment of wheelset axle and loading pattern

According to the adopted designations the coordinate of the design cross-section in the loading pattern is:

$$
x=l_{1}-0.5 B .
$$


According to the formula (6) test-bench operational force is:

$$
F=\frac{\pi d_{x}^{3}}{32 x} \sigma_{-1},
$$

where $d_{x}=d_{1}-$ design cross-section diameter (location of fillet on the box journal; see Fig. 4); $\sigma_{-1}=145 \mathrm{MPa}-$ axle fatigue strength in the design cross-section [12].

Lumping together ( $\left.6^{*}\right)$ and (9), we will obtain:

$$
F=\frac{\pi d_{x}^{3}}{32\left(l_{1}-0.5 B\right)} \sigma_{-1} \text {. }
$$

For the test example $F=274.4 \mathrm{kN}$.

From the formula (7) the debalance characteristic me (product of debalance weight and its eccentricity) is:

$$
m e=\frac{F}{\omega^{2}} \text {. }
$$

The calculation is performed for three values of angular speed of debalance rotation provided the direct drive is used (without multiplying gear). For the test example we obtain the following characteristics of rebalances:

$$
\begin{aligned}
& -n_{1}=3000 \mathrm{rpm} ; \omega_{1}=314 \mathrm{~s}^{-1} ; m e=2.78 \mathrm{~kg} \cdot \mathrm{m} ; \\
& -n_{2}=1500 \mathrm{rpm} ; \omega_{2}=157 \mathrm{~s}^{-1} ; m e=11.1 \mathrm{~kg} \cdot \mathrm{m} ; \\
& -n_{3}=1000 \mathrm{rpm} ; \omega_{3}=105 \mathrm{~s}^{-1} ; m e_{1}=24.9 \mathrm{~kg} \cdot \mathrm{m} .
\end{aligned}
$$

The use of lower values $\omega$ leads to excessive duration of testing.

Let's consider three types of debalances (Fig. 7):
a) Rectangular;
b) Circular;
c) Sectoral.
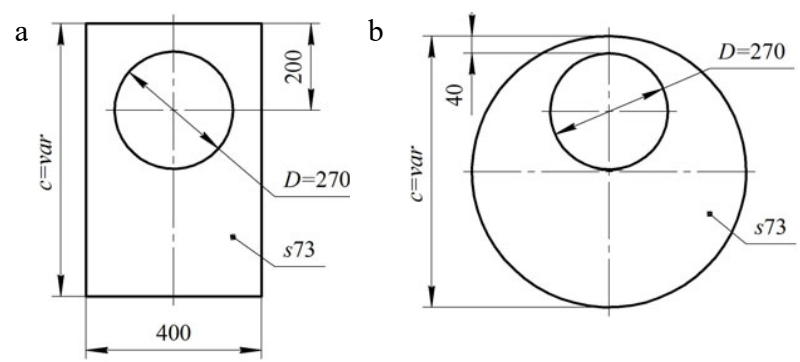

Fig. 7. Types of debalances (dimensions - in $\mathrm{mm}$ ): a - Rectangular; b - Circular; $\mathrm{c}-$ Sectoral

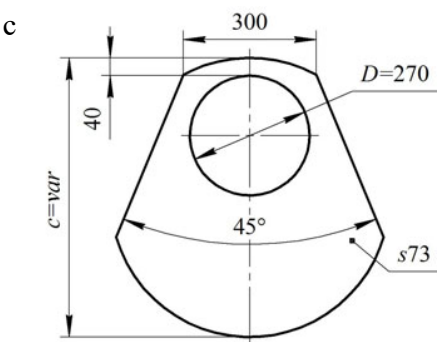

Let's adopt that thickness of each debalance is identical and equals to the width of the bearing (for the test example $s=B=73 \mathrm{~mm}$ ).

Multiple-option 3D-simulation of debalances with varying of the value $c(c=v a r$, Fig. 7$)$ allowed to obtain the following dependencies ( $m-$ in $\mathrm{kg} ; e-$ in $\mathrm{m})$ :

- For rectangular debalance

$$
m(e)=379 e+54.77 \text {; }
$$

- For circular debalance

$$
m(e)=705 e^{2}+242 e+21.6 ;
$$

- For sectoral debalance

$$
m(e)=877 e^{2}+338 e+20.8 .
$$

Graphic representation of functions (12)-(14) and (15)-(17) in the same coordinate plane allows to define the weight $m$ and eccentricity $e$ of each type of debalances that ensure the required value of the testbench operational force. Values $m$ and $e$ are coordinates of the intersection points of relevant curves (Fig. 8). The results of the plot analysis are presented in Table 1-3.

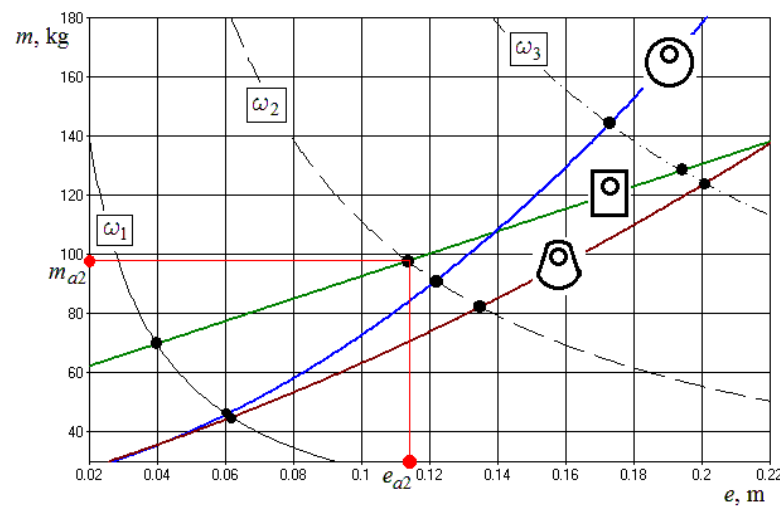

Fig. 8. Plot for determination of weight and eccentricity of the debalance

Table 1. Parameters of Rectangular debalance.

\begin{tabular}{|c|c|c|c|}
\hline$\omega, \mathrm{s}^{-1}$ & $m, \mathrm{~kg}$ & $e, \mathrm{~m}$ & $I, \mathrm{~kg} \cdot \mathrm{m}^{2}$ \\
\hline$\omega_{1}=314$ & 69.9 & 0.037 & 2.86 \\
\hline$\omega_{2}=157$ & 97.2 & 0.114 & 5.89 \\
\hline$\omega_{3}=105$ & 128.0 & 0.191 & 12.23 \\
\hline
\end{tabular}

Table 2. Parameters of Circular debalance

\begin{tabular}{|c|c|c|c|}
\hline$\omega, \mathrm{s}^{-1}$ & $m, \mathrm{~kg}$ & $e, \mathrm{~m}$ & $I, \mathrm{~kg} \cdot \mathrm{m}^{2}$ \\
\hline$\omega_{1}=314$ & 46.3 & 0.060 & 1.54 \\
\hline$\omega_{2}=157$ & 90.7 & 0.119 & 4.89 \\
\hline$\omega_{3}=105$ & 144.9 & 0.171 & 12.00 \\
\hline
\end{tabular}

Table 3. Parameters of Sectoral debalance.

\begin{tabular}{|c|c|c|c|}
\hline$\omega, \mathrm{s}^{-1}$ & $m, \mathrm{~kg}$ & $e, \mathrm{~m}$ & $I, \mathrm{~kg} \cdot \mathrm{m}^{2}$ \\
\hline$\omega_{1}=314$ & 44.5 & 0.064 & 1.47 \\
\hline$\omega_{2}=157$ & 81.6 & 0.133 & 4.42 \\
\hline$\omega_{3}=105$ & 125.2 & 0.198 & 10.78 \\
\hline
\end{tabular}

Let's determine the power consumed for debalance rotation using the formula

$$
E=0.5 I \omega^{2}
$$

Results of calculations are summarized in Table 4. 
Table 4. Power consumption for debalance rotation.

\begin{tabular}{|c|c|c|c|}
\hline \multirow{2}{*}{$\omega, \mathrm{s}^{-1}$} & \multicolumn{3}{|c|}{$E, \mathrm{~kJ}$, for debalance } \\
\cline { 2 - 4 } & rectangular & circular & sectoral \\
\hline$\omega_{1}=314$ & 141.0 & 75.9 & 72.5 \\
\hline$\omega_{2}=157$ & 72.6 & 60.3 & 54.6 \\
\hline$\omega_{3}=105$ & 67.4 & 66.2 & 59.4 \\
\hline
\end{tabular}

As it is obvious, the lowest are power consumption for sectoral debalance rotation at the angular speed of $\omega_{2}=157 \mathrm{~s}^{-1}$.

The studies showed that further increasing of the debalance rotation frequency leads to a decrease of its weight, eccentricity and moment of inertia. However, due to high angular speeds of debalance rotation the magnitude of the test-bench operational force begins to be influenced by inaccuracies of fabrication and mounting of wheelset axle and vibrator elements. Thus, when using (in vibrator design) the motor with shaft rotation frequency of $n \approx 3000 \mathrm{rpm}$ and multiplying gear with the ratio $u \approx 1 / 15$ we will obtain $\omega=4710 \mathrm{~s}^{-1}$. Under such conditions the eccentricity of sectoral debalance $e=5.9 \cdot 10^{-4} \mathrm{~m}$, that by the order of magnitude concurs with the wheelset axle runout tolerance.

By analyzing the results obtained we come to the conclusion that by the criterion of power consumption the rational is the sectoral debalance rotating at the angular speed of around $150 \mathrm{~s}^{-1}$. Therewith, the debalance weight is around $80 \mathrm{~kg}$, and the eccentricity is $135 \mathrm{~mm}$. Under such conditions the criterion specified gains the lowest value.

\section{Conclusions}

1. For the purpose of conducting the bench testing to determine the railway wheelset axle fatigue strength the rational is the "cantilever beam" loading pattern, which make the rationality criteria (operational forces, power consumption and test-bench overall dimension lengthwise) to gain the lowest values.

2. The test-bench operational force depends on wheelset axle dimensions (diameter and box journal length), therefore the universal test-bench has to provide the possibility of varying the operational force depending on axle nominal size.

3 . By the criteria of design complexity and power consumption the best one is the vibrator that ensures the test-bench operational force application by rotating the imbalance weight (debalance) around the rigid wheelset axle. In case of using it the criteria used gain the smallest magnitude.
4. For testing the axles for wheelsets of the considered size, by criterion of power consumption the rational is the vibrator design with sectoral debalance rotating at the angular speed of around $150 \mathrm{~s}^{-1}$. Therewith, the debalance weight is around $80 \mathrm{~kg}$, and the eccentricity is $135 \mathrm{~mm}$. Under such conditions the criterion specified gains the lowest value.

\section{References}

1. M. Novosad, R. Fajkoš, B. Řeha, R. Řezníček. Procedia Engineering 2, 2259-2268 (2010). https://doi.org/10.1016/j.proeng.2010.03.242

2. A. Răduţă, C. Locovei, M. Nicoară, L. Cucuruz. WSEAS Transactions on Applied and Theoretical Mechanics 5, 197-207 (2010).

3. M. Filippini, M. Luke, I. Varfolomeev, D. Regazzi, S. Beretta. Structural Integrity Procedia 4, 11-18 (2017). https://doi.org/10.1016/j.prostr.2017.07.013

4. S. Raksha, P. Anofriev, V. Bohomaz, O. Kuropiatnyk Naukovyi Visnyk Natsionalnoho Hirnychoho Universytetu 2, 108-115 (2019). https://doi.org/10.29202/nvngu/2019-2/16

5. S. Myamlin, J. Kalivoda, L. Neduzha. Procedia Engineering 187, 688-695 (2010). https://doi.org/10.1016/j.proeng.2017.04.439

6. S. Raksha, O. Kuropiatnyk, P. Anofriev, D. Onopreychuk, I. Kovalov. MATEC Web of Conferences 230, $01010 \quad$ (2018). https://doi.org/10.1051/matecconf/201823001010

7. GOST 31373. Wheelsets of locomotives and motorpowered units. Strength calculations and tests (2008).

8. SU 1076816. Ustanovka dlia ispytaniia osei kolesnykh par na ustalost pri krugovom isgibe. A. Adiklis, et. al. (1984).

9. RU 2650327. Stend dlia ispytaniia koles $i$ osei kolesnykh par na soprotivlenie ustalosti. E. Kochetkov, et. al. (2018).

10. RU 2651629. Stend dlia ispytaniia koles $i$ osei kolesnykh par na soprotivlenie ustalosti $i$ sposob provedeniia ispytanii. A. Bidulia, et. al. (2018).

11. GOST 33200. Axles for wheelsets of railway rolling stock. General specifications. (2014).

12. GOST 33783. Wheelsets of the railway rolling stock. Methods of strength performance determination. (2016). 